Case Report

\title{
Complete Heart Block Complicating Takotsubo Syndrome: Case Report and Literature Review
}

\author{
Michael J. McGee $\mathbb{D}^{1,2,3}$ William Yu, ${ }^{2}$ Joshua McCarthy, ${ }^{2}$ Malcolm Barlow, ${ }^{2}$ \\ and Rosemary Hackworthy ${ }^{2}$ \\ ${ }^{1}$ School of Medicine and Public Health, University of Newcastle, Newcastle, NSW, Australia \\ ${ }^{2}$ Cardiovascular Department, John Hunter Hospital, Newcastle, NSW, Australia \\ ${ }^{3}$ Hunter Medical Research Institute, Newcastle, NSW, Australia
}

Correspondence should be addressed to Michael J. McGee; michael.mcgee@health.nsw.gov.au

Received 30 March 2020; Revised 1 June 2020; Accepted 7 July 2020; Published 19 August 2020

Academic Editor: Manabu Shirotani

Copyright (c) 2020 Michael J. McGee et al. This is an open access article distributed under the Creative Commons Attribution License, which permits unrestricted use, distribution, and reproduction in any medium, provided the original work is properly cited.

An 81-year-old woman presents with shortness of breath resulting in a diagnosis of picornavirus and complete heart block. Troponin was elevated and there was concern about acute coronary syndrome. The final diagnosis after echocardiogram and coronary angiogram was Takotsubo syndrome in addition to the heart block which required pacemaker insertion.

\section{History of Presentation}

An 81-year-old woman presented to a peripheral emergency department with a two-week history of shortness of breath. She had been commenced on oral antibiotics for a lower respiratory tract infection in the community.

1.1. Past Medical History. The patient has a history of hypertension, dyslipidaemia, osteoporosis, anxiety, and gastrooesophageal reflux disease.

1.2. Medications at Admission. Irbesartan $300 \mathrm{mg}$ daily, hydrochlorothiazide $12.5 \mathrm{mg}$ daily, thyroxine $50 \mathrm{mcg}$ daily, rosuvastatin $10 \mathrm{mg}$ daily, sertraline $25 \mathrm{mg}$ daily, aspirin $100 \mathrm{mg}$ daily, metoprolol $12.5 \mathrm{mg}$ twice daily were the medications for the patients.

1.3. Clinical Course. On assessment in the emergency department, the patient was found to be in complete heart block $(\mathrm{CHB})$ with a ventricular escape of $48 \mathrm{bpm}$ (Figure 1$)$. She denied chest pain on presentation or recently. High sensitivity troponin was found to be elevated at $525 \mathrm{ng} / \mathrm{L}(<16)$. The patient was transported to the local tertiary hospital for the management of acute coronary syndrome and complete heart block. A respiratory viral PCR swab was sent prior to transport. The swab later returned positive for picornavirus. B-type natriuretic peptide (BNP) was elevated at $1174 \mathrm{ng} / \mathrm{L}$ $(<266)$ and subsequent troponins fell.

Echocardiogram after transfer revealed a large area of left ventricular apical hypokinesis, moderate atrial dilatation, and tricuspid regurgitation in the context of the known complete heart block (Figure 2 and supplementary figure 1).

Cardiac catheterisation was delayed secondary to inability to lie flat as a result of the respiratory infection, with cough fits and agitation without desaturation. This was performed on day 9 postpresentation. The coronary angiogram revealed nonobstructive coronary artery disease, with a focal $50 \%$ stenosis in the midleft circumflex coronary artery (supplemental figure 2). The left ventriculogram performed revealed near normalisation of the apical hypokinesis and an elevated end diastolic pressure of $20 \mathrm{mmHg}$ (Figure 3). Laboratory findings are listed in Table 1.

1.4. Management. The complete heart block continued after cessation of the metoprolol. The patient was initially managed conservatively as the ventricular escape was greater than $40 \mathrm{bpm}$ without hypotension. The patient did have 


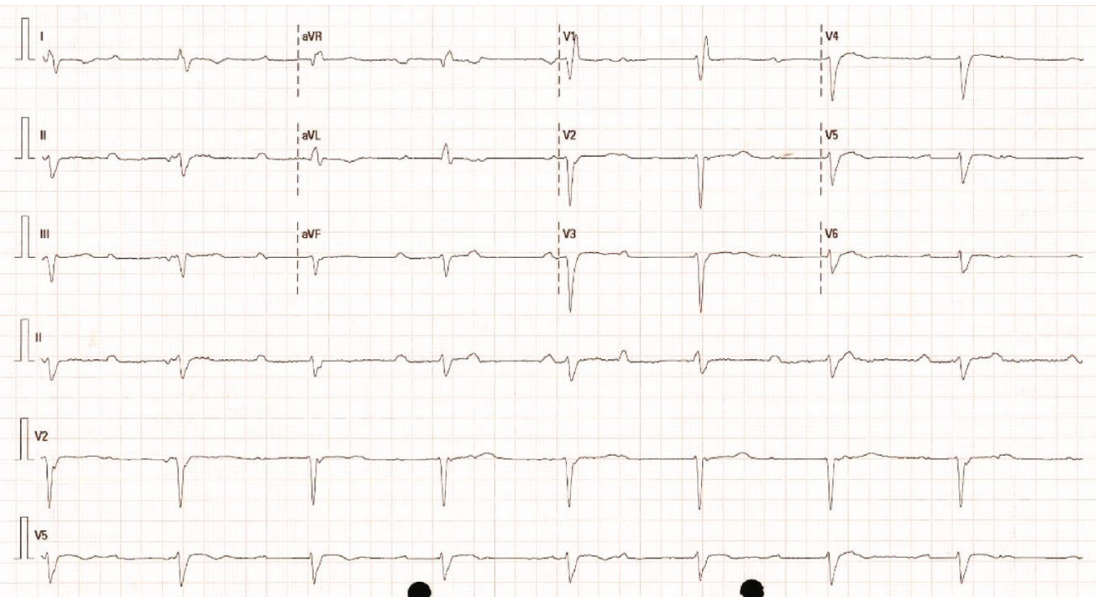

FigURE 1: Electrocardiogram revealing complete heart block with a ventricular escape at approximately 48 beats per minute.

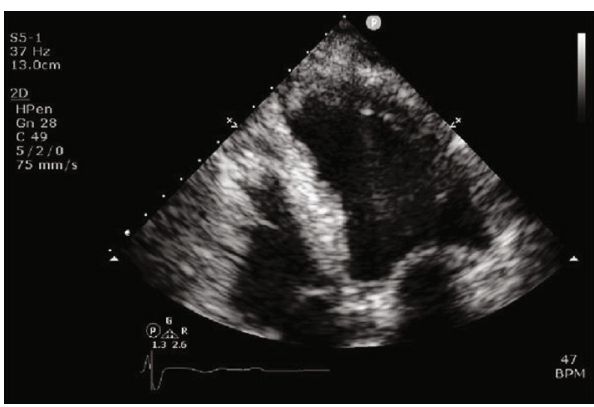

(a)

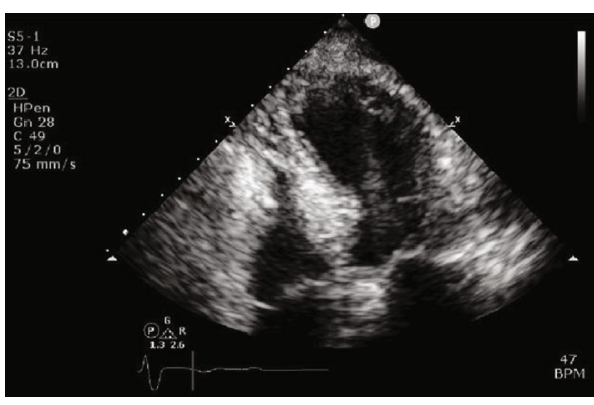

(b)

Figure 2: Transthoracic echocardiogram performed on presentation. Apical 4 chamber view, at end diastole (a) and end systolic (b) demonstrating left ventricular apical hypokinesis.

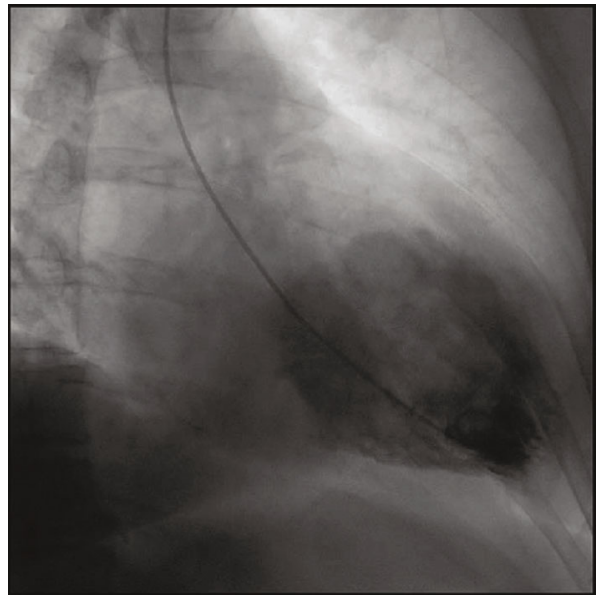

(a)

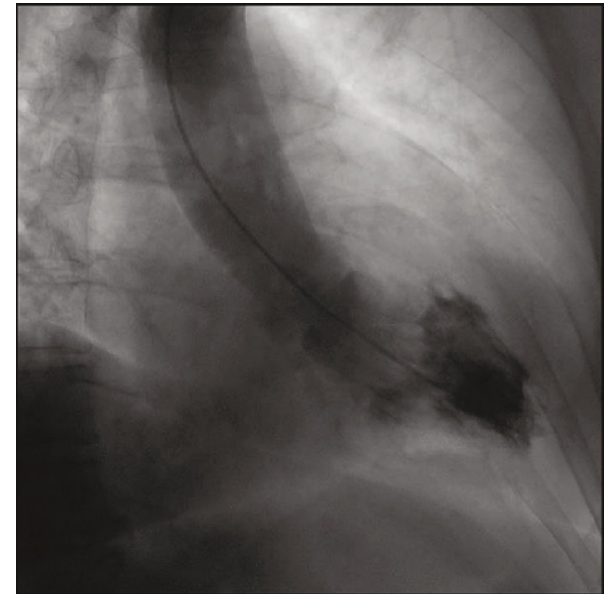

(b)

FIGURE 3: Cardiac catheterisation performed on day 9, left ventriculogram at end diastole (a) and end systole (b) demonstrating resolution of the apical hypokinesis.

exacerbations of coughing and agitation when lying flat, which could be attributed to either heart failure or respiratory tract infection. Chest X-ray on presentation was not consistent with heart failure despite the raised BNP. The picornavirus infection was managed conservatively. Nine days after presentation, once the patient was able to lie flat without incident, a dual chamber Biotronik pacemaker was implanted, DDD 60-130 (Figure 4). The patient was discharged on aspirin, irbesartan, rosuvastatin, and sertraline.

1.5. Follow-Up. Interrogation of the pacemaker 6 weeks postimplantation revealed underlying complete heart block with 
TABLE 1

\begin{tabular}{lccc}
\hline Value & Initial & Peak & Reference range \\
\hline Troponin I & 525 & 525 & $<16 \mathrm{ng} / \mathrm{L}$ \\
TSH & 2.84 & & $0.40-5.00 \mathrm{mIU} / \mathrm{L}$ \\
Sodium & 131 & 139 & $135-145 \mathrm{mmol} / \mathrm{L}$ \\
Potassium & 4.4 & 5.5 & $3.5-5.2 \mathrm{mmol} / \mathrm{L}$ \\
Creatinine & 82 & 121 & $45-90 \mu \mathrm{mol} / \mathrm{L}$ \\
Calcium (corrected) & 2.56 & 2.71 & $2.10-2.60 \mathrm{mmol} / \mathrm{L}$ \\
BNP & 1174 & & $<266 \mathrm{ng} / \mathrm{L}$ \\
CRP & 38 & 49 & $<5 \mathrm{mg} / \mathrm{L}$ \\
WCC & 11.5 & 14.1 & $4.0-11.0 \times 10^{9} / \mathrm{L}$ \\
Haemoglobin & 123 & 131 & $115-165 \mathrm{~g} / \mathrm{L}$ \\
\hline
\end{tabular}

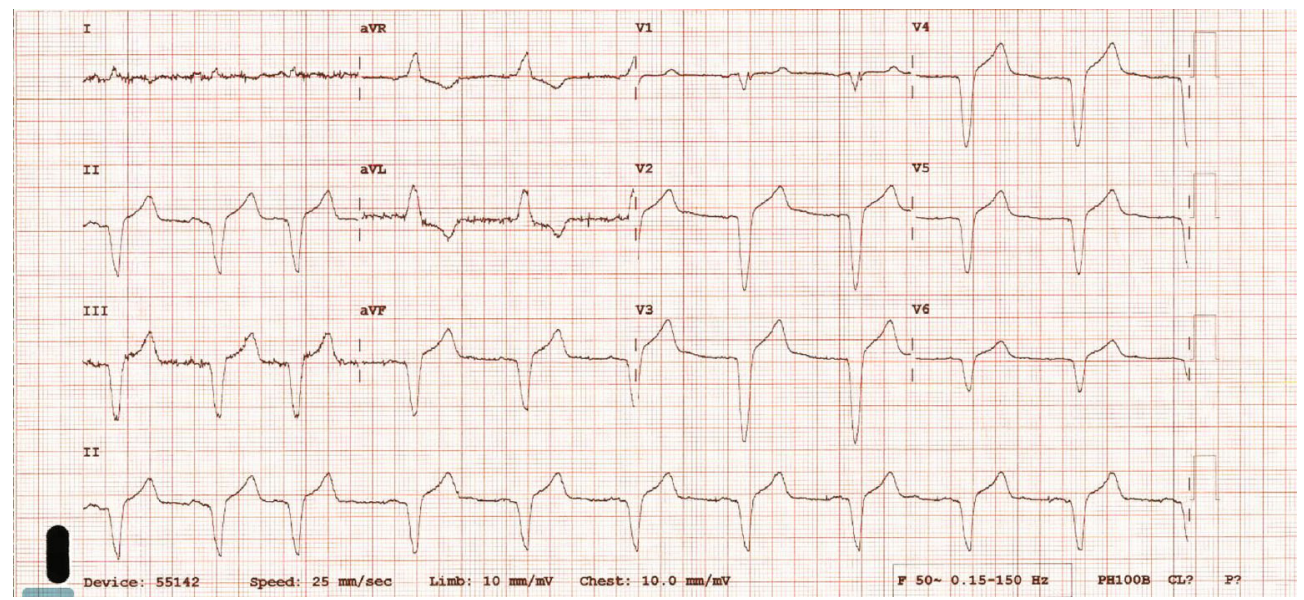

FIGURE 4: Electrocardiogram following implantation of a dual chamber pacemaker with atrial sensed, ventricular paced complexes.

$100 \%$ ventricular pacing, no tachyarrhythmias, and satisfactory device parameters. The patient remains independent in the community 6 months following discharge.

\section{Discussion}

The patient had previously been referred to a cardiologist as an outpatient due to concerns regarding cardiomegaly on chest $\mathrm{X}$-ray and resistant hypertension. Investigations completed 6 months prior to the patient's hospitalisation revealed normal left ventricular size and systolic function on an echocardiogram and right bundle branch block on an electrocardiogram.

It appears that the Takotsubo syndrome (TTS) in our patient's case was precipitated by the lower respiratory tract infection with picornavirus. This phenomenon of an emotional or physiological insult as a trigger for TTS has been well described. This case is unusual given the presence of complete heart block in association with TTS at time of presentation. It is unclear when the patient developed complete heart block as the ventricular escape was robust and was well tolerated hemodynamically. There was preexisting right bundle branch block, and the possibility of progressive conduction disease is high.
The alternative diagnoses for this case include acute coronary syndrome and viral-induced myocarditis. Picornavirus family has been associated with viral myocarditis in other cases and series [1]. Cardiac magnetic resonance imaging could help distinguish in cases were the diagnosis is unclear. We felt the characteristics of the echocardiogram and angiogram were consistent with TTS, and subsequently, cardiac magnetic resonance imaging and cardiac biopsy were not pursued.

Acute coronary syndrome was another possible diagnosis, with spontaneous recanalization. We felt this was unlikely given that the extensive wall motion abnormality was beyond a single coronary artery territory (the patient did not have a left anterior descending that wrapped around the apex) and was disproportionate to the peak troponin.

Several case reports of TTS and CHB exist [2-4]. The clinical challenges are twofold: firstly, determining which precipitates the other, and secondly, determining the need for a cardiac implantable electronic device (CIED). If the TTS is secondary to $\mathrm{CHB}$, then pacing will be required; however, if the CHB is secondary to TTS, then there is the possibility of recovery and a CIED can be avoided. Cases of both scenarios have been reported in the literature $[5,6]$.

CIED implantation is not a benign procedure with recent research documenting a high complication rate of 
TABLE 2

\begin{tabular}{|c|c|c|c|c|c|c|c|c|}
\hline Case & Year & Author & Age & Sex & TTS and CHB & CIED & F/U period (months) & Resolved \\
\hline 1 & 2004 & Saito et al. [12] & 86 & $\mathrm{~F}$ & 1 & Yes & NR & Unknown \\
\hline 2 & 2006 & Nef et al. [13] & 58 & $\mathrm{M}$ & 1 & Yes & 3 & Yes \\
\hline 3 & 2006 & Lee et al. [14] & 72 & $\mathrm{~F}$ & 1 & No & $<1$ & Yes \\
\hline 4 & 2007 & Nault et al. [2] & 62 & $\mathrm{~F}$ & 1 & Yes & 24 & Yes \\
\hline 5 & \multirow{2}{*}{2008} & \multirow{2}{*}{ Kurisu et al. [9] } & 78 & M & 1 & Yes & $<1$ & Unknown \\
\hline 6 & & & 87 & $\mathrm{~F}$ & 1 & Yes & NR & Unknown \\
\hline 7 & 2009 & Inoue et al. [3] & 82 & $\mathrm{~F}$ & 1 & Yes & 3 & Unknown \\
\hline 8 & \multirow{2}{*}{2009} & \multirow{2}{*}{ Kodama et al. [15] } & 57 & $\mathrm{~F}$ & 1 & Yes & NR & Unknown \\
\hline 9 & & & 39 & $\mathrm{~F}$ & 1 & Yes & NR & Unknown \\
\hline 10 & 2011 & Siry et al. [16] & 70 & $\mathrm{~F}$ & 1 & Yes & NR & Unknown \\
\hline 11 & 2012 & Benouda et al. [17] & 81 & $\mathrm{~F}$ & 1 & Yes & NR & Unknown \\
\hline 12 & 2012 & Shanmugasundar et al. [4] & 72 & $\mathrm{~F}$ & 1 & Yes & NR & Unknown \\
\hline 13 & 2013 & Chadha et al. [18] & 61 & $\mathrm{~F}$ & 1 & No & 12 & Yes \\
\hline 14 & 2013 & Wakiya et al. [19] & 86 & $\mathrm{~F}$ & 1 & Yes & 12 & No \\
\hline 15 & 2013 & Sugiura et al. [5] & 63 & $\mathrm{~F}$ & 1 & No & NR & Unknown \\
\hline 16 & \multirow{4}{*}{2015} & \multirow{4}{*}{ Stiermaier et al. [6] } & NR & NR & 1 & Yes & NR & Unknown \\
\hline 17 & & & NR & NR & 1 & Yes & NR & Unknown \\
\hline 18 & & & NR & NR & 1 & Yes & NR & Unknown \\
\hline 19 & & & NR & NR & 1 & No & NR & Unknown \\
\hline 20 & 2015 & Korantzopoulos et al. [20] & 71 & $\mathrm{~F}$ & 1 & Yes & 6 & No \\
\hline 21 & 2017 & Inayat et al. [11] & 59 & $\mathrm{~F}$ & 1 & Yes & 6 & No \\
\hline 22 & 2018 & Afzal et al. [21] & 62 & $\mathrm{~F}$ & 1 & Yes & 6 & No \\
\hline 23 & 2019 & Sakul et al. [10] & 73 & $\mathrm{~F}$ & 1 & Yes & 20 & No \\
\hline 24 & 2020 & McGee & 81 & $\mathrm{~F}$ & 1 & Yes & 3 & No \\
\hline
\end{tabular}

NR: not reported.

approximately 10\% [7]. There are case reports of TTS occurring post CIED implantation [8]. Case reports of TTS and $\mathrm{CHB}$ have described implantation of dual chamber pacemakers, biventricular pacemakers, and defibrillator for the treatment of $\mathrm{CHB}$ [9-11]. One of the largest consecutive series of TTS reported a CHB prevalence of $2.2 \%$ [6]. Including our case, we were able to identify 24 reported cases of CHB complicating TTS. In four of the cases, a CIED was not implanted due to the resolution of the heart block. In further two cases, the AV block resolved postimplantation of the CIED (Table 2).

\section{Conclusion}

TTS is not a benign condition and presents a number of clinical challenges. An association with complete heart block is not uncommon and often requires CIED implantation.

Take-home messages and learning objectives are as follows:

(i) Takotsubo syndrome can result in a wide range of dangerous arrhythmias, both brady and tachycardic

(ii) Takotsubo syndrome can complicate other cardiovascular disease entities (iii) Most, but not all patients who have complete heart block and Takotsubo syndrome require a pacemaker

\section{Abbreviations}

CIED: Cardiac implantable electronic device

CHB: Complete heart block (third-degree atrioventricular block)

TTS: Takotsubo syndrome.

\section{Disclosure}

During the time of drafting, all authors were employed by Hunter New England Health. The employer had no role in drafting or reviewing the manuscript.

\section{Conflicts of Interest}

The authors declare that they have no conflicts of interest.

\section{Supplementary Materials}

Supplementary 1. Supplemental Figure 1: Transthoracic echocardiogram performed on presentation. Apical 2 chamber view, at end diastole (left) and end systolic (right) demonstrating left ventricular apical hypokinesis. Supplemental 
figure 2: Coronary angiography, with the left coronary artery (left) and the right coronary artery (right) demonstrating non-obstructive coronary artery disease.

Supplementary 2. Supplemental Videos: Video 1: Transthoracic echocardiogram performed on presentation. Apical 2 chamber view demonstrating apical hypokinesis with preserved basal function consistent with takotsubo syndrome. Video 2: Transthoracic echocardiogram performed on presentation. Apical 3 chamber view demonstrating apical hypokinesis with preserved basal function consistent with takotsubo syndrome. Video 3: Transthoracic echocardiogram performed on presentation. Apical 4 chamber view demonstrating apical hypokinesis with preserved basal function consistent with takotsubo syndrome. Video 4: Cardiac catheterisation performed on day 9, left ventriculogram demonstrating resolution of the apical hypokinesis.

\section{References}

[1] E. Arbustini, E. Porcu, O. Bellini et al., "Enteroviral infection causing fatal myocarditis and subclinical myopathy," Heart, vol. 83, no. 1, pp. 86-90, 2000.

[2] M. A. Nault, A. Baranchuk, C. S. Simpson, and D. P. Redfearn, "Takotsubo cardiomyopathy: a novel "proarrhythmic" disease," Anadolu Kardiyoloji Dergisi, vol. 7, pp. 101-103, 2007.

[3] M. Inoue, H. Kanaya, T. Matsubara, Y. Uno, T. Yasuda, and K. Miwa, "Complete atrioventricular block associated with Takotsubo cardiomyopathy," Circulation Journal, vol. 73, no. 3, pp. 589-592, 2009.

[4] R. Shanmugasundaram, K. Tamilarasu, G. Rajendiran, and A. Murali, "An uncommon presentation of a rare disease high-degree AV block with Takotsubo cardiomyopathy," Indian Heart Journal, vol. 64, no. 5, pp. 511-514, 2012.

[5] T. Sugiura, Y. Dohi, S. Yamashita et al., "Midventricular ballooning Takotsubo cardiomyopathy complicated by transient complete atrioventricular block," Internal Medicine, vol. 52, no. 17, pp. 1919-1921, 2013.

[6] T. Stiermaier, C. Eitel, S. Denef et al., "Prevalence and clinical significance of life-threatening arrhythmias in Takotsubo cardiomyopathy," Journal of the American College of Cardiology, vol. 65, no. 19, pp. 2148-2150, 2015.

[7] I. Ranasinghe, C. Labrosciano, D. Horton et al., "Institutional variation in quality of cardiovascular implantable electronic device implantation: a cohort study," Annals of Internal Medicine, vol. 171, no. 5, pp. 309-317, 2019.

[8] S. G. Chun, V. Kwok, D. K. Pang, and T. K. Lau, "Transient left ventricular apical ballooning syndrome (Takotsubo cardiomyopathy) as a complication of permanent pacemaker implantation," International Journal of Cardiology, vol. 117, no. 1, pp. e27-e30, 2007.

[9] S. Kurisu, I. Inoue, T. Kawagoe et al., "Torsade de pointes associated with bradycardia and Takotsubo cardiomyopathy," The Canadian Journal of Cardiology, vol. 24, no. 8, pp. 640-642, 2008.

[10] N. F. N. Sakul, S. Shrestha, N. Balabbigari, and S. Talati, "Complete heart block: a rare complication of Takotsubo syndrome," Case Reports in Cardiology, vol. 2019, Article ID 2576373, 5 pages, 2019.
[11] F. Inayat, H. U. H. Virk, W. Ullah, and I. Riaz, "Takotsubo cardiomyopathy-related complete heart block and torsades de pointes," BML Case Reports, vol. 2017, 2017.

[12] M. Saito, Y. Hayashi, O. Sasaki, M. Inoue, S. Fujioka, and M. Kouno, "A case of foxhole-shaped left ventricular abnormality induced by complete atrioventricular block with delayed recovery of wall motion," Nihon Ronen Igakkai Zasshi., vol. 41, no. 5, pp. 546-551, 2004.

[13] H. M. Nef, H. Möllmann, J. Sperzel et al., "Temporary thirddegree atrioventricular block in a case of apical ballooning syndrome," International Journal of Cardiology, vol. 113, no. 2, pp. e33-e35, 2006.

[14] W. L. Lee, L. F. Miao, H. W. Chan, and M. Z. Chen, "Takotsubo syndrome with transient complete atrioventricular block," Chinese Medical Journal, vol. 119, no. 1, pp. 73-76, 2006.

[15] S. Kodama, K. Miyoshi, Y. Shiga et al., "Takotsubo cardiomyopathy complicated by high-grade atrioventricular block: a report of two cases," Experimental and Clinical Cardiology, vol. 14, no. 2, pp. e35-e38, 2009.

[16] M. Siry, N. Scheffold, D. Wimmert-Roidl, and G. Konig, "A rare complication of Takotsubo cardiomyopathy," $D M W$ Deutsche Medizinische Wochenschrift, vol. 136, no. 4, pp. 129-132, 2011.

[17] L. Benouda, V. Roule, A. Foucault, Z. Dahdouh, A. Lebon, and P. Milliez, "Conduction disturbances in Takotsubo cardiomyopathy: a cause or a consequence?," International Journal of Cardiology, vol. 159, no. 1, pp. 61-62, 2012.

[18] S. Chadha, A. Lodha, V. Shetty, A. Sadiq, G. Hollander, and J. Shani, "Complete heart block in Takotsubo cardiomyopathy," Heart \& Lung, vol. 42, no. 1, pp. 48-50, 2013.

[19] M. Wakiya, H. Hara, M. Iwano et al., "Complete atrioventricular block associated with not apical but midventricular ballooning," Journal of Cardiology Cases, vol. 7, no. 4, pp. e109e113, 2013.

[20] K. Panagiotis, N. Nikas Dimitrios, L. Konstantinos et al., "High-grade atrioventricular block and Takotsubo cardiomyopathy: case report and review of the literature," International Cardiovascular Research Journal, vol. 9, no. 2, article e11407, 2017.

[21] A. Afzal, J. Watson, J. W. Choi, J. M. Schussler, and M. D. Assar, "Takotsubo cardiomyopathy in the setting of complete heart block," Baylor University Medical Center Proceedings, vol. 31, no. 4, pp. 502-505, 2018. 